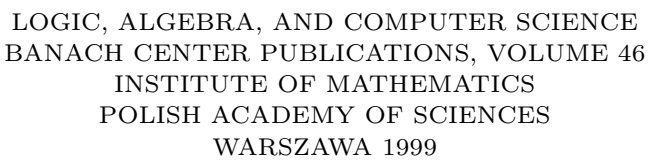

\title{
A CONSTRUCTIVE PROOF THAT EVERY 3-GENERATED $\ell$-GROUP IS ULTRASIMPLICIAL
}

\author{
DANIELE MUNDICI \\ Department of Computer Science \\ University of Milano \\ Via Comelico 39-41 \\ 20135 Milano, Italy \\ E-mail:mundici@imiucca.csi.unimi.it \\ GIOVANNI PANTI \\ Department of Mathematics \\ University of Udine \\ Via delle Scienze 208 \\ 33100 Udine, Italy \\ E-mail:panti@dimi.uniud.it
}

\begin{abstract}
We discuss the ultrasimplicial property of lattice-ordered abelian groups and their associated MV-algebras. We give a constructive proof of the fact that every lattice-ordered abelian group generated by three elements is ultrasimplicial.
\end{abstract}

1. Preliminaries. The infinite-valued calculus of Łukasiewicz stands to MV-algebras as the classical two-valued calculus stands to boolean algebras. Indeed, as proved by Chang [Cha58], the latter coincide with the subclass of MV-algebras satisfying the equation $x \oplus x=x$. The ultrasimplicial property of MV-algebras is a generalization of the fundamental fact that every boolean algebra is generated by the limit of the direct system of its finite partitions. Accordingly, this property is a prerequisite for such results as the joint refinability of MV-algebraic partitions, or even - assuming the appropriate $\sigma$-closure conditions - for the definition of an MV-algebraic notion of entropy.

Using the categorical equivalence between MV-algebras and abelian lattice-ordered groups with strong unit (see [Mun86]), one has a natural counterpart of the ultrasimplicial

1991 Mathematics Subject Classification: 03B50, 20K05.

The first named author is partially supported by CNR-GNSAGA Project on Symbolic Computation.

The paper is in final form and no version of it will be published elsewhere.

$[169]$ 
property for any such group $G$-with or without strong unit - to the effect that every finite set of positive elements of $G$ is positively generated by an independent set $B$ of positive elements (see [Ell79] and [Han83]).

In [Mun88], from the algebraic analysis of disjunctive normal forms in the infinitevalued calculus, it is proved that every free abelian lattice-group is ultrasimplicial. Thus, since Handelman proved that the ultrasimplicial property is preserved under formation of quotients by order-ideals [Han83, Theorem 3(ii)], it follows that every abelian latticegroup, as well as every MV-algebra, is ultrasimplicial. However, Handelman's proof is nonconstructive, and gives no hint on how to construct the independent set $B$.

Aim of this paper is to give a constructive proof, for the case of 3 -generated latticegroups, corresponding to 2-generated MV-algebras. We shall work throughout in the framework of partially ordered groups.

2. Definitions. A partially ordered abelian group is an abelian group $G$, together with a submonoid $G^{+}$of $G$, such that $G^{+}$generates $G$ as a group and $G^{+} \cap\left(-G^{+}\right)=\{0\}$. A

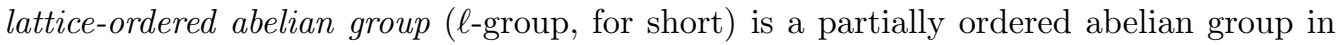
which the order induced by

$$
a \leq b \quad \text { iff } \quad b-a \in G^{+}
$$

is a lattice order. Equivalently, an $\ell$-group is a structure $(G,+,-, 0, \vee, \wedge)$ such that $(G,+,-, 0)$ is an abelian group, $(G, \vee, \wedge)$ is a lattice, and + distributes over $\vee$ and $\wedge$. The words $\ell$-subgroup and $\ell$-homomorphism have their standard universal algebraic meaning, with respect to the signature $(+,-, 0, \vee, \wedge)$. An $\ell$-ideal is an $\ell$-subgroup $J$ of $G$ which is convex in $G$ (i.e., $a \leq b \leq c$ in $G$ and $a, c \in J$ imply $b \in J$ ). Thus, $\ell$-ideals are precisely the same as kernels of $\ell$-homomorphisms.

An $\ell$-group is simplicially ordered iff it is isomorphic to a finite power $\mathbb{Z}^{m}$ of the integers, with componentwise order. A partially ordered abelian group $G$ is ultrasimplicially ordered iff it can be expressed as the union of an increasing chain

$$
G_{0} \subseteq G_{1} \subseteq G_{2} \subseteq \cdots
$$

of simplicially ordered groups, satisfying $G_{i}^{+} \subseteq G_{i+1}^{+}$for every $i$. As shown in [Han83, Proposition 1], the ultrasimplicial property has the following equivalent reformulation:

(U) for every $a_{1}, \ldots, a_{r} \in G^{+}$there exist $b_{1}, \ldots, b_{s} \in G^{+}$that are linearly independent over $\mathbb{Z}$ and $\operatorname{span} a_{1}, \ldots, a_{r}$ with integer coefficients $\geq 0$.

It is well known that the free $\ell$-group over $n$ generators is the $\ell$-subgroup $\mathrm{F} \ell(n)$ of $\mathbb{R}^{\left(\mathbb{R}^{n}\right)}$ generated by the projection functions $\mathbf{x}_{i}: \mathbb{R}^{n} \rightarrow \mathbb{R}$, for $i=1, \ldots, n$. Equivalently, $\mathrm{F} \ell(n)$ is the $\ell$-group of all homogeneous piecewise-linear functions with integer coefficients. These are defined as follows: a homogeneous piecewise-linear function with integer coefficients (a hpli function, for short) is a continuous function $\mathbf{f}: \mathbb{R}^{n} \rightarrow \mathbb{R}$ for which there exist finitely many elements $\mathbf{f}_{1}, \ldots, \mathbf{f}_{m}$ of $\operatorname{Hom}\left(\mathbb{Z}^{n}, \mathbb{Z}\right)$ such that, for every $u \in \mathbb{R}^{n}$, there exists an index $i$ with $\mathbf{f}(u)=\mathbf{f}_{i}(u)$.

By [Mun88], every free $\ell$-group is ultrasimplicial. In [Han83, Theorem 3(ii)], it is claimed that the ultrasimplicial property is preserved under quotients, but the proof is - to say the least - nonconstructive. Let us note that Elliott's proof [Ell79] that every 
totally ordered group is ultrasimplicial provides also a recipe for constructing, given $a_{1}, \ldots, a_{r} \in G^{+}$, elements $b_{1}, \ldots, b_{s}$ satisfying property $(\mathrm{U})$. The same holds for the proof in [Mun88], which can also be extended to finitely presented $\ell$-groups (i.e., quotients of $\mathrm{F} \ell(n)$ by congruences generated by finitely many equations of the form $\mathbf{f}=\mathbf{g}$, for $\mathbf{f}, \mathbf{g} \in \mathrm{F} \ell(n))$; details of this extension are spelled out in [MP93].

In this paper we give a direct, effective proof that every $\ell$-group generated by three elements is ultrasimplicial.

3. The main result. We need some preliminaries in piecewise-linear topology. Let $v_{1}, \ldots, v_{m} \in \mathbb{Z}^{n}$; the polyhedral cone $\sigma$ generated by $v_{1}, \ldots, v_{m}$ is their positive hull. Explicitly:

$$
\sigma=\left\langle v_{1}, \ldots, v_{m}\right\rangle=\left\{r_{1} v_{1}+\cdots+r_{m} v_{m}: r_{1}, \ldots, r_{m} \in \mathbb{R}^{+}\right\},
$$

where $\mathbb{R}^{+}$is the set of real numbers $\geq 0$. We say that $\sigma$ is $k$-dimensional iff the linear space spanned by $\sigma$ is $k$-dimensional. We set

$$
\sigma^{\vee}=\left\{\mathbf{f} \in \operatorname{Hom}\left(\mathbb{R}^{n}, \mathbb{R}\right): \mathbf{f}(u) \geq 0 \text {, for every } u \in \sigma\right\} .
$$

A face $\tau$ of $\sigma$ is any set of the form $\tau=\sigma \cap \operatorname{ker} \mathbf{f}$, for some $\mathbf{f} \in \sigma^{\vee}$. Equivalently, a face of $\sigma$ is a nonempty convex subset $\tau$ of $\sigma$ such that every line segment in $\sigma$ which has an interior point in $\tau$ lies entirely in $\tau$. The relative interior rel int $\sigma$ of $\sigma$ is the topological interior of $\sigma$ relative to the $\mathbb{R}$-vector space generated by $\sigma$. If $\sigma$ is $k$-dimensional, then $u \in$ rel int $\sigma$ iff there exist vectors $v_{1}, \ldots, v_{k} \in \sigma$ that are linearly independent over $\mathbb{R}$ and such that $u=a_{1} v_{1}+\cdots+a_{k} v_{k}$, with $a_{1}, \ldots, a_{k} \in \mathbb{R}^{+} \backslash\{0\}$ (see, e.g., [Ful93, §1.2]).

A simplicial cone is a polyhedral cone of the form $\left\langle v_{1}, \ldots, v_{m}\right\rangle$, with $v_{1}, \ldots, v_{m}$ linearly independent over $\mathbb{R}$. A nonzero point $v \in \mathbb{Z}^{n}$ is primitive iff its coordinates are relatively prime. The vertices of a simplicial cone $\sigma$ are the uniquely determined primitive points $v_{1}, \ldots, v_{m}$ such that $\sigma=\left\langle v_{1}, \ldots, v_{m}\right\rangle$.

A unimodular cone is a simplicial cone of the form $\left\langle v_{1}, \ldots, v_{m}\right\rangle$, where $v_{1}, \ldots, v_{m} \in \mathbb{Z}^{n}$ and there exist $v_{m+1}, \ldots, v_{n} \in \mathbb{Z}^{n}$ such that $v_{1}, \ldots, v_{m}, v_{m+1}, \ldots, v_{n}$ constitute a free basis for $\mathbb{Z}^{n}$. A fan is a finite set $\Sigma$ of polyhedral cones such that:

(i) every face of every cone of $\Sigma$ belongs to $\Sigma$;

(ii) any two cones of $\Sigma$ intersect in a common face.

The union of all cones of $\Sigma$ is denoted by $|\Sigma| ; \Sigma$ is a complete fan iff $|\Sigma|=\mathbb{R}^{n}$. If all cones of $\Sigma$ are unimodular, then $\Sigma$ is said to be unimodular. Complete unimodular fans correspond to nonsingular projective toric varieties [Oda88], [Ful93], [Ewa96].

If $\Sigma, \Delta$ are fans, $|\Sigma|=|\Delta|$, and every cone of $\Delta$ is contained in some cone of $\Sigma$, then we say that $\Delta$ is a subdivision of $\Sigma$, and we write $\Delta \leq \Sigma$. In this case every cone of $\Sigma$ is a union of cones of $\Delta$. Any vertex of any cone of $\Sigma$ is a vertex of $\Sigma$.

Lemma 3.1. Let $\Sigma$ be a fan, $u \in|\Sigma|$. Then there exists $\sigma \in \Sigma$ such that $u \in \operatorname{rel}$ int $\sigma$ and, for every $\sigma \neq \sigma^{\prime} \in \Sigma$, we have $u \notin$ relint $\sigma^{\prime}$.

Proof. Let $\sigma=\bigcap\{\tau \in \Sigma: u \in \tau\}$. Any polyhedral cone is the disjoint union of the relative interior of its faces [Oda88, Appendix]. This applies to $\sigma$, and hence $u \in \operatorname{rel}$ int $\sigma$. 
Let $\sigma=\left\langle u, v, w_{1}, \ldots, w_{r}\right\rangle$ be an $(r+2)$-dimensional unimodular cone. Then $\tau=\langle u, v\rangle$ is a face of $\sigma$; we call $w=u+v$ the Farey mediant of $\tau$. Let $\sigma^{\prime}=\left\langle w, v, w_{1}, \ldots, w_{r}\right\rangle$, $\sigma^{\prime \prime}=\left\langle u, w, w_{1}, \ldots, w_{r}\right\rangle$. Assume that $\Sigma$ is a unimodular fan, with $\sigma \in \Sigma$. Define a subdivision $\Sigma^{\prime}$ of $\Sigma$ by replacing each cone $\sigma \in \Sigma$ of which $\tau$ is a face by the two cones $\sigma^{\prime}, \sigma^{\prime \prime}$ obtained as above, along with all their faces. Then $\Sigma^{\prime}$ is a unimodular fan, and we say that $\Sigma^{\prime}$ is obtained by starring $\Sigma$ along $\tau$ (see [Oda88, Proposition 1.26], or [Ewa96, Definition 6.1]).

Let $u$ be a vertex of the complete unimodular fan $\Sigma$. The Schauder hat $\mathbf{u}$ (or $\mathbf{u}_{\Sigma}$, if we need to make explicit the dependence upon $\Sigma$ ) of $\Sigma$ at $u$ is the unique homogeneous piecewise-linear function $\mathbf{u}: \mathbb{R}^{n} \rightarrow \mathbb{R}$ such that

(i) $\mathbf{u}(u)=1$;

(ii) $\mathbf{u}(v)=0$, for every vertex $v \neq u$ of $\Sigma$;

(iii) $\mathbf{u}$ is homogeneous linear on each cone of $\Sigma$.

Schauder hats exist for arbitrary vertices of $\Sigma$; indeed, the unimodularity of the cones of $\Sigma$ automatically ensures that the coefficients of every linear piece of $\mathbf{u}$ are integers (see [Mun88, Proposition 1.3] for details). Hence $\mathbf{u}$ is a hpli function, and an element of $\mathrm{F} \ell(n)$.

Let $\Sigma$ be a complete unimodular fan in $\mathbb{R}^{n}$; let $u_{1}, \ldots, u_{t}$ display all vertices of $\Sigma$. Then the Schauder hats $\mathbf{u}_{1}, \ldots, \mathbf{u}_{t}$ are linearly independent over $\mathbb{Z}$; let $H_{\Sigma}$ be the subgroup of $\mathrm{F} \ell(n)$ generated - using the group operations only- by $\mathbf{u}_{1}, \ldots, \mathbf{u}_{t}$. Note that $H_{\Sigma}$, with the order inherited from $\mathrm{F} \ell(n)$, is $\ell$-isomorphic to $\mathbb{Z}^{t}$ as a simplicial group, but it is not an $\ell$-subgroup of $\mathrm{F} \ell(n)$, since the lattice operations are different in the two structures. The zero-set of $\mathbf{f} \in \mathrm{F} \ell(n)$ is $Z \mathbf{f}=\left\{v \in \mathbb{R}^{n}: \mathbf{f}(v)=0\right\}$. Let a fixed $\ell$-ideal $J$ of $\mathrm{F} \ell(n)$ be given; define

$$
Z \Sigma=\bigcap\left\{Z \mathbf{f}: \mathbf{f} \in H_{\Sigma} \cap J\right\} .
$$

The dependence of $Z \Sigma$ on $J$ is tacitly understood.

Lemma 3.2. For any $\mathbf{f} \in H_{\Sigma}$, we have $\mathbf{f} \in J$ iff $Z \mathbf{f} \supseteq Z \Sigma$.

Proof. For the nontrivial direction, suppose $Z \mathbf{f} \supseteq Z \Sigma$. Since $H_{\Sigma} \cap J$ is a subgroup of the finitely generated free $\mathbb{Z}$-module $H_{\Sigma}$, we can find generators $\mathbf{g}_{1}, \ldots, \mathbf{g}_{h}$ of $H_{\Sigma} \cap J$. Then

$$
Z \Sigma=Z \mathbf{g}_{1} \cap \cdots \cap Z \mathbf{g}_{h} \subseteq Z \mathbf{f} .
$$

Let $\mathbf{g}=\left|\mathbf{g}_{1}\right|+\cdots+\left|\mathbf{g}_{h}\right| \in J$. Since $Z \mathbf{g} \subseteq Z|\mathbf{f}|$, we have by compactness $0 \leq|\mathbf{f}| \leq m \mathbf{g}$ in $\mathrm{F} \ell(n)$, for some multiple $m \mathbf{g}$ of $\mathbf{g}$. Since $J$ is convex, $|\mathbf{f}| \in J$, and since $-|\mathbf{f}| \leq \mathbf{f} \leq|\mathbf{f}|$, we have $\mathbf{f} \in J$.

LEMmA 3.3. Let $\sigma$ be a $k$-dimensional cone of $\Sigma$, and let $v_{1}, \ldots, v_{p} \in \sigma \cap Z \Sigma$. Let $V$ denote the $\mathbb{R}$-vector space spanned by $v_{1}, \ldots, v_{p}$. Then $\sigma \cap Z \Sigma \supseteq \sigma \cap V$.

Proof. Every $\mathbf{f} \in H_{\Sigma}$ is homogeneous linear on $\sigma$.

LEMMA 3.4. If $\Delta$ is a unimodular subdivision of $\Sigma$, then $Z \Delta \subseteq Z \Sigma$.

Proof. $H_{\Sigma} \subseteq H_{\Delta}$, and hence $H_{\Sigma} \cap J \subseteq H_{\Delta} \cap J$. 
LEMMA 3.5. Let $\Sigma$ be such that $Z \Sigma$ is a union of cones of $\Sigma$. Then the set

$$
\{\mathbf{u} / J: \mathbf{u} \text { is a Schauder hat of } \Sigma \text { and } \mathbf{u} \notin J\}
$$

is linearly independent over $\mathbb{Z}$ in $\mathrm{F} \ell(n) / J$.

Proof. Let $\mathbf{f}=a_{1} \mathbf{u}_{1}+\cdots+a_{t} \mathbf{u}_{t}$, where $a_{1}, \ldots, a_{t} \in \mathbb{Z}$ and $\mathbf{u}_{1}, \ldots, \mathbf{u}_{t}$ are Schauder hats of $\Sigma$ at distinct vertices of $\Sigma$. Assume $a_{1} \neq 0, \mathbf{u}_{1} \notin J$ : we will show that $\mathbf{f} \notin J$. Since $u_{1} \notin J$, by Lemma 3.2 there exists $v \in Z \Sigma \backslash Z \mathbf{u}_{1}$. Let $\sigma$ be the cone of $\Sigma$ to whose relative interior $v$ belongs; as $v \in Z \Sigma$, and $Z \Sigma$ is a union of cones of $\Sigma$, we have $\sigma \subseteq Z \Sigma$. Since $\mathbf{u}_{1}$ is not identically 0 on $\sigma, u_{1}$ must be a vertex of $\sigma$. Since $\mathbf{f}\left(u_{1}\right)=a_{1} \mathbf{u}_{1}\left(u_{1}\right)=a_{1} \neq 0$ then $Z \mathbf{f} \nsupseteq Z \Sigma$, and hence $\mathbf{f} \notin J$.

TheOrem 3.6. Let $\Sigma$ be a complete unimodular fan, $|\Sigma|=\mathbb{R}^{3}$. Let $J$ be an $\ell$-ideal of $\mathrm{F} \ell(3)$. Then there exists a subdivision $\Delta$ of $\Sigma$ with the following two properties:

(i) $\Delta$ can be obtained from $\Sigma$ via a finite sequence of starrings along 2-dimensional cones;

(ii) $Z \Delta$ is a union of cones of $\Delta$.

4. Proof of Theorem 3.6. Let $\sigma \in \Sigma, k \in\{2,3\}, 1 \leq j \leq k$. We say that $\sigma$ is of type $(k, j)$ with respect to $\Sigma$ iff the following hold:

(1) $\sigma$ is $k$-dimensional;

(2) $\sigma \cap Z \Sigma$ is $j$-dimensional;

(3) rel int $\sigma \cap Z \Sigma \neq \emptyset$.

If either rel int $\sigma \cap Z \Sigma=\emptyset$, or $\sigma$ is 0- or 1-dimensional, then $\sigma$ is of no type. Note that (3) is equivalent to:

$\left(3^{\prime}\right) \sigma \cap Z \Sigma$ is not contained in a proper face of $\Sigma$.

Indeed, (3) clearly implies $\left(3^{\prime}\right)$, while the reverse direction follows from Lemma 3.3.

Definition 4.1. For every $\sigma \in \Sigma$ of type $(k, j)$, and every unordered pair $u, v$ of distinct vertices of $\sigma$, we define the badness of $(\sigma, u, v)$, denoted by $\operatorname{bad}_{\Sigma}(\sigma, u, v)$, as follows:

(a) if $k=j=3$, or $k=j=2$, then $\operatorname{bad}_{\Sigma}(\sigma, u, v)=\infty$.

(b) if $k=3$ and $j=2$, then there exists a unique (up to multiplication by -1 ) primitive linear functional $\mathbf{f} \in \operatorname{Hom}\left(\mathbb{Z}^{3}, \mathbb{Z}\right)$ such that $\sigma \cap Z \mathbf{f}=\sigma \cap Z \Sigma$. Set

$$
\operatorname{bad}_{\Sigma}(\sigma, u, v)= \begin{cases}0, & \text { if } \mathbf{f}(u) \cdot \mathbf{f}(v) \geq 0 \\ |\mathbf{f}(u)|+|\mathbf{f}(v)|, & \text { otherwise. }\end{cases}
$$

(c) if $j=1$, then there exists a unique primitive $w \in \mathbb{Z}^{3}$ such that $\sigma \cap Z \Sigma=\langle w\rangle$. $w \in \operatorname{rel} i n t \sigma$, and can be written uniquely as a linear combination of the vertices of $\sigma$ with integer coefficients $>0$. Set $\operatorname{bad}_{\Sigma}(\sigma, u, v)=a+b$, where $a, b$ are the coefficients of $u, v$ in the above expression for $w$.

We shall use induction on eight parameters, ordered lexicographically from left to right as follows:

$$
s_{0}(3,3), s_{1}(3,2), s_{0}(3,2), s_{1}(3,1), s_{0}(3,1), s_{0}(2,2), s_{1}(2,1), s_{0}(2,1) .
$$


These parameters are defined by:

- $s_{0}(k, k)=$ number of cones of type $(k, k)$;

- for $j<k, s_{1}(k, j)=\sup \left\{\operatorname{bad}_{\Sigma}(\sigma, u, v): \sigma\right.$ is of type $\left.(k, j)\right\}$;

- for $j<k, s_{0}(k, j)=$ number of triples $(\sigma, u, v)$ such that $\sigma$ is of type $(k, j)$ and $\operatorname{bad}_{\Sigma}(\sigma, u, v)=s_{1}(k, j)$ (triples are unordered, so $\left.(\sigma, u, v)=(\sigma, v, u)\right)$.

Note that, for $j<k$, we have $s_{1}(k, j)=0$ iff $s_{0}(k, j)=0$ iff $\Sigma$ contains no cones of type $(k, j)$.

LEMma 4.2. If, for every $1 \leq j<k \in\{2,3\}, \Sigma$ contains no cones of type $(k, j)$, then $Z \Sigma$ is a union of cones of $\Sigma$.

Proof. Let $u \in Z \Sigma$, and let $\sigma$ be the cone of $\Sigma$ to whose relative interior $u$ belongs. If $\sigma$ is $k$-dimensional, then by our assumption $\sigma \cap Z \Sigma$ must be $k$-dimensional, too. By Lemma 3.3, $\sigma \subseteq Z \Sigma$.

We equip types with the following order:

$$
(3,3) \succ(3,2) \succ(3,1) \succ(2,2) \succ(2,1) \succ \text { no type. }
$$

LEMMA 4.3. Let $\Sigma, \Delta$ be complete unimodular fans, and assume that $\Delta$ is a subdivision of $\Sigma$. Let $\delta \in \Delta$, and let $\sigma=\bigcap\{\tau \in \Sigma: \delta \subseteq \tau\}$. Then the type of $\delta$ in $\Delta$ is less than or equal to the type of $\sigma$ in $\Sigma$. If $\delta=\sigma$ and the two types are equal, then $\operatorname{bad}_{\Delta}(\sigma)=\operatorname{bad}_{\Sigma}(\sigma)$.

Proof. Clear from the definitions.

Lemma 4.4. Suppose $\Sigma$ contains a cone of type $(k, j)$, with $1 \leq j<k \in\{2,3\}$; let $s_{0}(3,3), \ldots, s_{0}(2,1)$ be the parameters associated to $\Sigma$. Then there exists a starring of $\Sigma$ along a 2-dimensional cone such that-letting $\Sigma^{\prime}$ be the resulting fan and $s_{0}^{\prime}(3,3), \ldots, s_{0}^{\prime}(2,1)$ its associated parameters - the 8-tuple $\left(s_{0}^{\prime}(3,3), \ldots, s_{0}^{\prime}(2,1)\right)$ is strictly smaller than $\left(s_{0}(3,3), \ldots, s_{0}(2,1)\right)$ in the lexicographic ordering.

Proof. Let $(k, j)$ be the largest type (with respect to $\succ$ ) such that $s_{1}(k, j) \neq 0$. Let $\sigma \in \Sigma$ be of type $(k, j)$, and let $u, v$ be vertices of $\sigma \operatorname{with}_{\operatorname{bad}_{\Sigma}}(\sigma, u, v)=s_{1}(k, j)$. We star $\Sigma$ along $\tau=\langle u, v\rangle$, obtaining $\Sigma^{\prime}$; let $w=u+v . \tau$ is the intersection of two uniquely determined 3-dimensional cones $\lambda, \mu \in \Sigma$. If $k=2$, then $\sigma=\tau$, while if $k=3$, we assume $\sigma=\lambda$. Upon taking a section of these cones by a suitable plane we obtain the following picture:

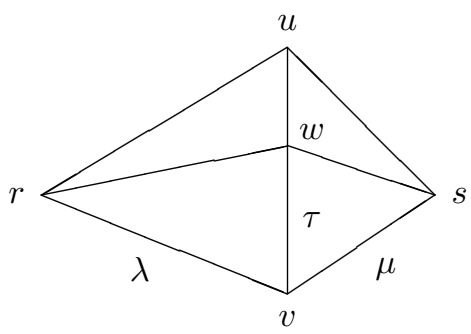

The starring replaces:

- $\lambda$ with $\lambda^{\prime}=\langle u, w, r\rangle, \lambda^{\prime \prime}=\langle w, v, r\rangle$, and $\langle w, r\rangle$; 
- $\tau$ with $\tau^{\prime}=\langle u, w\rangle, \tau^{\prime \prime}=\langle w, v\rangle$, and $\langle w\rangle$;

- $\mu$ with $\mu^{\prime}=\langle u, w, s\rangle, \mu^{\prime \prime}=\langle w, v, s\rangle$, and $\langle w, s\rangle$.

We proceed arguing by cases:

Case 1. $k=3, j=2, \sigma=\lambda$. Then $\sigma \cap Z \Sigma=\sigma \cap Z \mathbf{f}$, for some primitive $\mathbf{f} \in \operatorname{Hom}\left(\mathbb{Z}^{3}, \mathbb{Z}\right)$. Without loss of generality, $\mathbf{f}(u)<0$. Also, $\tau \cap Z \Sigma=\tau \cap Z \mathbf{f}=\langle p\rangle$, with $p \in \operatorname{rel}$ int $\tau$; by Lemmas 3.3 and 4.3 , each of $\mu, \lambda^{\prime}, \lambda^{\prime \prime}, \mu^{\prime}, \mu^{\prime \prime}$ is of type $\preceq(3,2)$. By Lemma 4.3 , the parameter $s_{0}(3,3)$ cannot increase. If it decreases, we are through. Otherwise, it suffices to prove that, for every $\rho \in\left\{\lambda^{\prime}, \lambda^{\prime \prime}, \mu^{\prime}, \mu^{\prime \prime}\right\}$, if $\rho$ is of type $(3,2), u^{\prime}, v^{\prime}$ are vertices of $\rho$, and $\left\langle u^{\prime}, v^{\prime}\right\rangle$ is not a cone of $\Sigma$, then $\operatorname{bad}_{\Sigma^{\prime}}\left(\rho, u^{\prime}, v^{\prime}\right)<\operatorname{bad}_{\Sigma}(\sigma, u, v)$. To this purpose, we again distinguish:

Subcase 1.1. $\rho=\lambda^{\prime}, u^{\prime}=u, v^{\prime}=w$. By Lemma 4.3, together with the assumption that $\lambda^{\prime}$ is of type $(3,2)$, in $\Sigma^{\prime}$, we have $\lambda^{\prime} \cap Z \Sigma^{\prime}=\lambda^{\prime} \cap Z \mathbf{f}$. If $p \notin \operatorname{rel} \operatorname{int}\langle u, w\rangle$, then $\operatorname{bad}_{\Sigma^{\prime}}\left(\rho, u^{\prime}, v^{\prime}\right)=0$. Otherwise, $\mathbf{f}(u)<0<\mathbf{f}(w)<\mathbf{f}(v)$, and $\operatorname{bad}_{\Sigma^{\prime}}\left(\rho, u^{\prime}, v^{\prime}\right)=|\mathbf{f}(u)|+$ $|\mathbf{f}(w)|<|\mathbf{f}(u)|+|\mathbf{f}(v)|=\operatorname{bad}_{\Sigma}(\sigma, u, v)$.

Subcase 1.2. $\rho=\lambda^{\prime}, u^{\prime}=r, v^{\prime}=w$. Again, $\lambda^{\prime} \cap Z \Sigma^{\prime}=\lambda^{\prime} \cap Z \mathbf{f}$. We may assume $\mathbf{f}(r) \cdot \mathbf{f}(w)<0$ for, otherwise, $\operatorname{bad}_{\Sigma^{\prime}}\left(\lambda^{\prime}, r, w\right)=0$. Either $\mathbf{f}(u)<0<\mathbf{f}(w)<\mathbf{f}(v)$, or $\mathbf{f}(u)<\mathbf{f}(w)<0<\mathbf{f}(v)$. In the first case, $\operatorname{bad}_{\Sigma^{\prime}}\left(\lambda^{\prime}, r, w\right)=|\mathbf{f}(r)|+|\mathbf{f}(w)|<|\mathbf{f}(r)|+|\mathbf{f}(v)|=$ $\operatorname{bad}_{\Sigma}(\sigma, r, v) \leq \operatorname{bad}_{\Sigma}(\sigma, u, v)$. In the second case, $\operatorname{bad}_{\Sigma^{\prime}}\left(\lambda^{\prime}, r, w\right)=|\mathbf{f}(r)|+|\mathbf{f}(w)|<$ $|\mathbf{f}(r)|+|\mathbf{f}(u)|=\operatorname{bad}_{\Sigma}(\sigma, r, u) \leq \operatorname{bad}_{\Sigma}(\sigma, u, v)$.

Subcase 1.3. $\rho=\mu^{\prime}, u^{\prime}=u, v^{\prime}=w$. Then $\mu$ is of type $(3,2)$ in $\Sigma$. Let $\mathbf{g} \in \operatorname{Hom}\left(\mathbb{Z}^{3}, \mathbb{Z}\right)$ be primitive satisfying $\mu \cap Z \mathbf{g}=\mu \cap Z \Sigma$; then $\mu^{\prime} \cap Z \mathbf{g}=\mu^{\prime} \cap Z \Sigma^{\prime}$ and $\mathbf{g}(p)=0$. If $p \notin \operatorname{rel} \operatorname{int}\langle u, w\rangle$, then $\operatorname{bad}_{\Sigma^{\prime}}\left(\mu^{\prime}, u, w\right)=0$. Otherwise, without loss of generality, $\mathbf{g}(u)<$ $0<\mathbf{g}(w)<\mathbf{g}(v)$, and $\operatorname{bad}_{\Sigma^{\prime}}\left(\mu^{\prime}, u, w\right)=|\mathbf{g}(u)|+|\mathbf{g}(w)|<|\mathbf{g}(u)|+|\mathbf{g}(v)|=\operatorname{bad}_{\Sigma}(\mu, u, v) \leq$ $s_{1}(3,2)$.

Subcase 1.4. $\rho=\mu^{\prime}, u^{\prime}=s, v^{\prime}=w$. Then arguing as for Subcase 1.2 one gets the desired conclusion.

Subcase 1.5. $\rho=\lambda^{\prime \prime}$, or $\rho=\mu^{\prime \prime}$. The proof is the same as for the previous subcases.

Case 2. $k=3, j=1, \sigma=\lambda$. By our choice of $(k, j), \Sigma$ contains no cones of type $(3,2)$; by Lemmas 3.3 and 4.3 , each of $\mu, \lambda^{\prime}, \lambda^{\prime \prime}, \mu^{\prime}, \mu^{\prime \prime}$ is either of type $(3,1)$, or of no type. If either parameter $s_{0}(3,3), s_{1}(3,2), s_{0}(3,2)$ happens to change, then, by Lemma $4.3, s_{0}(3,3)$ must decrease - and we are done. If, on the other hand, none of these parameters changes, then to obtain the desired conclusion it suffices to prove that, for every $\rho \in\left\{\lambda^{\prime}, \lambda^{\prime \prime}, \mu^{\prime}, \mu^{\prime \prime}\right\}$, if $\rho$ is of type $(3,1), u^{\prime}, v^{\prime}$ are vertices of $\rho$, and $\left\langle u^{\prime}, v^{\prime}\right\rangle \notin \Sigma$, then $\operatorname{bad}_{\Sigma^{\prime}}\left(\rho, u^{\prime}, v^{\prime}\right)<$ $\operatorname{bad}_{\Sigma}(\sigma, u, v)$. We only consider the case $\rho=\lambda^{\prime}$, the other cases being similar. If $\rho=\lambda^{\prime}$, then $\sigma \cap Z \Sigma=\lambda^{\prime} \cap Z \Sigma^{\prime}=\langle p\rangle$, for a uniquely determined primitive $p \in \mathbb{Z}^{3}$. We have $p=a u+b v+c r$, for uniquely determined $a, b, c \in \mathbb{Z}^{+} \backslash\{0\}$. Since $p \in \operatorname{rel}$ int $\lambda^{\prime}$, we have $a>b$. Also, $p=(a-b) u+b w+c r$, uniquely. Since $\left\langle u^{\prime}, v^{\prime}\right\rangle \notin \Sigma$, we may assume $v^{\prime}=w$. If $u^{\prime}=u$, then $\operatorname{bad}_{\Sigma^{\prime}}\left(\lambda^{\prime}, u^{\prime}, v^{\prime}\right)=(a-b)+b<a+b=\operatorname{bad}_{\Sigma}(\sigma, u, v)$. If $u^{\prime}=r$, then $\operatorname{bad}_{\Sigma^{\prime}}\left(\lambda^{\prime}, u^{\prime}, v^{\prime}\right)=b+c<a+c=\operatorname{bad}_{\Sigma}(\sigma, u, r) \leq \operatorname{bad}_{\Sigma}(\sigma, u, v)$.

Case 3. $k=2, j=1, \sigma=\tau$. This is even simpler than Case 2. It suffices to observe that, by our choice of $(k, j), \Sigma$ contains no cones of either type $(3,2)$ or $(3,1)$. Also, each of $\lambda, \mu, \lambda^{\prime}, \lambda^{\prime \prime}, \mu^{\prime}, \mu^{\prime \prime}$ is of no type. In case one of the parameters $\succ s_{1}(2,1)$ happens 
to change, then, by Lemma 4.3, the greatest such changing parameter must actually decrease. If, on the other hand, no parameter $\succ s_{1}(2,1)$ does change, then the same argument as in Case 2 yields the desired conclusion.

The proof of Theorem 3.6 is now complete: as a matter of fact, let a complete unimodular fan $\Sigma$ be given, $|\Sigma|=\mathbb{R}^{3}$. If $Z \Sigma$ is not a union of cones of $\Sigma$, then, by Lemma $4.2, \Sigma$ contains a cone of type $(k, j)$, for some $j<k$. Using Lemma 4.4 , we have a chain of starrings $\Sigma>\Sigma^{\prime}>\Sigma^{\prime \prime}>\cdots$, which must terminate, since the set of 8-tuples of parameters is well ordered; say it stops at $\Delta$. Then $\Delta$ contains no cones of type $(k, j)$ for $k>j$, and Lemma 4.2 yields the desired conclusion.

\section{Conclusion}

THEOREM 5.1. Every $\ell$-group $G$ with three generators is ultrasimplicial.

Proof. We can safely identify $G$ with the quotient $\ell$-group $\mathrm{F} \ell(3) / J$, for some $\ell$ ideal $J$. Let $\mathbf{f}_{1} / J, \ldots, \mathbf{f}_{r} / J \in(\mathrm{F} \ell(3) / J)^{+}$. Replacing each $\mathbf{f}_{i}$ by $\mathbf{f}_{i} \vee \mathbf{0}$, and deleting all elements annihilated by the quotient map, we may assume $\mathbf{f}_{1}, \ldots, \mathbf{f}_{r} \in \mathrm{F} \ell(3)^{+} \backslash J$. Each $\mathbf{f}_{i}$ is of the form

$$
\mathbf{f}_{i}=\bigvee_{s \in S} \bigwedge_{t \in T} \mathbf{g}_{s t}^{i}
$$

where $S, T$ are finite index sets, and each $\mathbf{g}_{s t}^{i}$ is in $\operatorname{Hom}\left(\mathbb{Z}^{3}, \mathbb{Z}\right)$. Let $\mathbf{g}_{1}, \ldots, \mathbf{g}_{k}$ display all $\mathbf{g}_{s t}^{i}$, for $1 \leq i \leq r$. For every permutation $\varphi$ of $\{1, \ldots, k\}$, let

$$
\sigma_{\varphi}=\left\{x \in \mathbb{R}^{3}: \mathbf{g}_{\varphi(1)}(x) \leq \mathbf{g}_{\varphi(2)}(x) \leq \cdots \leq \mathbf{g}_{\varphi(k)}(x)\right\} .
$$

Then a routine argument shows that each $\sigma_{\varphi}$ is a polyhedral cone and that the set $\Gamma$ of all faces of all $\sigma_{\varphi}$ 's is a complete fan; moreover, every $\mathbf{f}_{i}$ is linear on every cone of $\Gamma$.

We make three successive refinements. The first two are standard constructions:

- firstly, we refine $\Gamma$ to a fan $\Pi$ whose cones are all simplicial. This can be accomplished without introducing new vertices, following [Ewa96, Theorem 4.2];

- secondly, we refine $\Pi$ to a complete unimodular fan $\Sigma$, as in the proof of [Ewa96, Theorem 8.5].

Thirdly, in the light of Theorem 3.6,

- we refine $\Sigma$ to a fan $\Delta$ such that $Z \Delta$ (relative to the ideal $J$ ) is a union of cones of $\Delta$.

Let $u_{1}, \ldots, u_{t}$ be the vertices of $\Delta$, and $\mathbf{u}_{1}, \ldots, \mathbf{u}_{t}$ their associated Schauder hats. Since, for every $i$,

$$
\mathbf{f}_{i}=\mathbf{f}_{i}\left(u_{1}\right) \cdot \mathbf{u}_{1}+\cdots+\mathbf{f}_{i}\left(u_{t}\right) \cdot \mathbf{u}_{t},
$$

it follows that the set $\left\{\mathbf{u}_{1} / J, \ldots, \mathbf{u}_{t} / J\right\} \backslash\{\mathbf{0} / J\}$ spans $\mathbf{f}_{1} / J, \ldots, \mathbf{f}_{r} / J$ positively and, by Lemma 3.5, is linearly independent over $\mathbb{Z}$ in $\mathrm{F} \ell(3) / J$. Hence condition (U) is satisfied, and the proof is complete.

Final remarks. A moment's reflection shows that a (constructive) proof of the ultrasimplicial property of all $n$-generated $\ell$-groups immediately extends to all $\ell$-groups. 
Thus it is natural to consider the following question: can the techniques of this paper be extended to $n$-generated $\ell$-groups ?

Theorem 3.6 is the only step in our proof that cannot be immediately generalized to higher dimensions. One can reasonably expect that the $n$-dimensional generalization of this theorem requires an induction argument over more complicated parameters. As a working hypothesis, for any $j$-dimensional cone $\sigma \cap Z \Sigma$ lying inside a $k$-dimensional cone $\sigma \in \Sigma$ natural badness parameters are provided by the Plücker coordinates of $\sigma \cap Z \Sigma$ with respect to the basis given by the $j$-dimensional faces of $\sigma$ (see, e.g., [BML67, Chapter XVI] for background). The attentive reader may have noticed that also the parameters used in this paper are based on Plücker coordinates, although in a slightly disguised form.

For an instructive example, let $\sigma=\left\langle v_{1}, \ldots, v_{4}\right\rangle$ be a 4 -dimensional cone of a fan $\Sigma$, and suppose the 2-dimensional cone $\sigma \cap Z \Sigma$ to be positively spanned by the two linearly independent vectors $p, q \in \mathbb{Z}^{n} \cap \sigma$. Let us construct the exterior algebra $\bigwedge^{2} \mathbb{Z}^{4}$, with basis $v_{1} \wedge v_{2}, \ldots, v_{3} \wedge v_{4}$, and assume that $p \wedge q$ has coordinates $\xi_{12}, \ldots, \xi_{34} \in \mathbb{Z}$ in $\wedge^{2} \mathbb{Z}^{4}$, with $\xi_{12}, \ldots, \xi_{34}$ relatively prime. It follows that

$$
s=\left|\xi_{12}\right|+\cdots+\left|\xi_{34}\right|
$$

is a natural badness parameter for $\sigma \cap Z \Sigma$ in $\sigma$. Indeed, $\sigma \cap Z \Sigma$ coincides with a face of $\sigma$ exactly when $s=1$. We must star $\sigma$ in such a way that $s$ decreases. Starring $\sigma$ along one of its faces corresponds to a base change in $\bigwedge^{2} \mathbb{Z}^{4}$. Now, while in dimension $\leq 3$ every base change corresponds to a starring, this does not hold in higher dimensions; for example, the base change arising from the substitution of $v_{1} \wedge v_{2}+v_{3} \wedge v_{4}$ for $v_{1} \wedge v_{2}$ does not correspond to any starring, because $v_{1} \wedge v_{2}+v_{3} \wedge v_{4}$ is not reducible in $\wedge^{2} \mathbb{Z}^{4}$. In algebraic-geometric terms, one has to figure out a path of starrings, leading from the base points $v_{1} \wedge v_{2}, \ldots, v_{3} \wedge v_{4}$ to the point $p \wedge q$, and never leaving the Grassmannian of lines in $\mathbb{P}^{3}$.

Further complications arise from the requirement that the starrings employed to decrease the badness of $\sigma \cap Z \Sigma$ in $\sigma$ should not result in increasing the badness of $\sigma^{\prime} \cap Z \Sigma$ in $\sigma^{\prime}$, for any $\sigma^{\prime} \in \Sigma$.

Closing a circle of ideas, as a final source of complication it might well be the case that the $n$-dimensional generalization of the results of this paper requires that the starring operation should be performed along arbitrary cones of $\Sigma$ (see [Oda88] or [Ewa96]), rather than only 2-dimensional cones.

\section{References}

[BML67] G. Birkhoff and S. Mac Lane, Algebra. The Macmillan Co., New York, 1967.

[Cha58] C. C. Chang, Algebraic analysis of many valued logics. Trans. Amer. Math. Soc., 88:467-490, 1958.

[El179] G. Elliote, On totally ordered groups, and $K_{0}$. In Ring Theory (Proc. Conf. Univ. Waterloo, Waterloo, 1978), volume 734 of Lecture Notes in Math., pages 1-49. Springer, 1979.

[Ewa96] G. Ewald, Combinatorial Convexity and Algebraic Geometry. Springer, 1996. 
[Ful93] W. Fulton, An introduction to Toric Varieties, volume 131 of Annals of Mathematics Studies. Princeton University Press, Princeton, N.J., 1993.

[Han83] D. Handelman, Ultrasimplicial dimension groups. Arch. Math., 40:109-115, 1983.

[MP93] D. Mundici and G. PAnTi, The equivalence problem for Bratteli diagrams. Technical Report 259, Department of Mathematics, University of Siena, Siena, Italy, 1993.

[Mun86] D. MundicI, Interpretation of AF $C^{*}$-algebras in Łukasiewicz sentential calculus. $J$. of Functional Analysis, 65:15-63, 1986.

[Mun88] D. Mundici, Farey stellar subdivisions, ultrasimplicial groups, and $K_{0}$ of $\mathrm{AF} C^{*}$ algebras. Advances in Math., 68(1):23-39, 1988.

[Oda88] T. OdA, Convex Bodies and Algebraic Geometry. Springer, 1988. 